\title{
Association of Comorbid Conditions with Six-month Survival and Disease Outcome in Patients of Necroinflammatory Otitis Externa
}

\author{
Khalid Cheema ${ }^{1}$, Nukhbatullah Awan ${ }^{1}$, Nadeem Raza ${ }^{1}$, Khadija Khan ${ }^{2}$, Samina Qamar ${ }^{3}$ and Sobia Ashraf ${ }^{3}$ \\ ${ }^{1}$ Department of ENT, King Edward Medical University, Lahore, Pakistan \\ ${ }^{2}$ Department of Obstetrics and Gynaecology, King Edward Medical University, Lahore, Pakistan \\ ${ }^{3}$ Department of Pathology, King Edward Medical University, Lahore, Pakistan
}

\begin{abstract}
Objective: To determine association of gender, causative organisms, control of diabetes, facial paralysis, infectious agent, and hearing loss with disease outcome, in terms of six-month improvement of symptoms, static condition or expiry of patients presenting with necroinflammatory otitis externa (NOE).

Study Design: Descriptive study.

Place and Duration of Study: ENT Department in collaboration with Pathology Department, KEMU/Mayo Hospital, Lahore from 2016 to 2019.

Methodology: Patients with NOE were inducted. Studied variables included age at presentation, gender, diabetes, glycated hemoglobin (HbAlc) levels, comorbid conditions, facial nerve involvement, hearing loss, CT and biopsy findings, and causative organisms; and their association with outcome was observed with significance at $p<0.05$.

Results: Out of 28 patients, there were 17 males (60.7\%) and 11 females (39.3\%). Association between gender and survival showed that $41.2 \%$ (7) males and $27.3 \%$ (3) females survived; and $23.5 \%$ (4) males and 1 (9.1\%) female expired within six months of diagnosis. Thinning of temporal bone $(2 / 5=40 \%)$ was common among the expired patients. Twenty percent $(1 / 5)$ patients diagnosed with squamous cell carcinoma and $80 \%(4 / 5)$ with granulation tissue (GT) expired $(p=0.543)$ All ten patients $(100 \%)$ that improved had mild to moderate hearing loss ( $p<0.001$ ). Among expired group, $80 \%(4 / 5)$ had HBAlc of more than 7 and $20 \%(1 / 5)$ had good control of diabetes. Aspergillus $(2 / 5=40 \%)$ and Pseudomonas $(1 / 5=20 \%)$ were the commonest among expired patients; Staphylococcus $(6 / 10=60 \%)$ and Pseudomonas $(3 / 10=30 \%)$ infections were more frequent among the survived $(p=0.005)$.

Conclusion: Previously pseudomonal infection was described as the only causative agent of NOE. This study showed a rising community-acquired disease with Staphylococcus aureus 6/10 (60\%) and Pseudomonas 3/10 (30\%) infection. Fungal infection is associated with poor survival and death, thus requiring aggressive management. Thinning of temporal bone on $\mathrm{CT}$, uncontrolled diabetes, sever hearing loss and facial paralysis score V/VI were associated with poor outcome of disease.
\end{abstract}

Key Words: Necroinflammatory otitis externa, Pseudomonas aeruginosa. Diabetes, Hearing loss, Facial paralysis, Temporal bone thinning, CT scan.

How to cite this article: Cheema K, Awan N, Raza N, Khan K, Qamar S, Ashraf S. Association of Comorbid Conditions with Six-month Survival and Disease Outcome in Patients of Necroinflammatory Otitis Externa. J Coll Physicians Surg Pak 2020; 30(05):498-502. DOI: https://doi.org/10.29271/jcpsp.2020.05.498.

\section{INTRODUCTION}

Necroinflammatory otitis externa (NOE) also known as malignant otitis externa (MOE) or skull-base osteitis (SBO) is a life-threatening infection of external auditory canal, os temporalis or base of skull, mostly seen in elderly and immunocompromised patients, particularly diabetics. ${ }^{1,2}$ Pathologically, it is divided into necrotizing otitis externa, in which only soft tissues and cartilage undergo necrosis, and osteomyelitis in which infection spreads to skull base bones and they undergo necrosis. ${ }^{3,4}$

Correspondence to: Dr. Samina Qamar, Department of Pathology, Room No. 6, King Edward Medical University, Lahore, Pakistan

E-mail:samnir3@gmail.com

Received: February 24, 2020; Revised: May 04, 2020;

Accepted: May 23, 2020

DOI: https://doi.org/10.29271/jcpsp.2020.05.498
Pseudomonas aeruginosa, which is an encapsulated, gram-negative rod, is the most common organism known to cause NOE. ${ }^{5}$ Others, like Staphylococcus aureus, Proteus mirabilis, Aspergillus fumigatus and Candida species have also been implicated. ${ }^{6}$ Many factors affect the disease course and outcome of NOE, e.g. age, duration and control of diabetes mellitus, HbAlc levels, granulation tissue in external auditory canal, involvement of cranial nerves, and the intracranial extent of infection assessed radiologically. The typical clinical presentation is severe unremitting otalgia which gets worsen at night and is refractory to conventional analgesics. ${ }^{7}$ Other signs such as aural fullness, foul smelling otorrhoea and hearing loss may also be present. NOE can be vigorously treated with appropriate antibiotics, while surgery is reserved for diagnostic purpose and local debridement. ${ }^{8}$

The condition needs vigilance and evidence-based management guidelines in the local context. This study aimed to determine the association of gender, causative organisms, control of diabetes, and hearing loss with prognosis, measured in terms of six-month improvement of symptoms or expiry of patients presenting with NOE. 


\section{METHODOLOGY}

It was a retrospective cohort study conducted in the Department of ENT in collaboration with Pathology Department of King Edward Medical University/Mayo Hospital, Lahore over a period of about four years from 2016 to 2019. Patients with MOE/NOE were divided into two groups: One obligatory and other occasional (Table I). Presence of obligatory criteria was considered essential for diagnosis of NOE and inclusion in the study. Twenty-eight (28) patients fulfilled the essential obligatory (major) criteria of NOE and had complete clinicoradiological and pathological data, required for the study. Patients with only minor signs of NOE and insufficient information were excluded. All enrolled patients had been admitted in the ward and managed adequately. Data was collected retrospectively through medical records of ward, in a structured questionnaire proforma which included demographic details, clinical features, findings on computed tomographic (CT) scan imaging, audiological assessment, culture and sensitivity results of aural secretions, facial paralysis (House Brackmann Score ${ }^{7}$ ), histopathology reports of ear biopsies, treatment, co-morbidities and outcome. Incisional biopsy was taken in all patients for the purpose of diagnosis; however, surgical debridement was reserved for patients with extensive disease to skull-base and adjacent areas. Patients were followed up for a period of six months.

\section{Table l: The diagnostic criteria of malignant otitis externa.}

\begin{tabular}{|l|}
\hline Major (obligatory) signs \\
\hline 1. Pain \\
2. Exudate \\
3. Edema \\
4. Granulations \\
5. Microabscesses \\
6. Positive Technetium-99 (99Tc) scan of failure of local treatment \\
$\quad$ after more than 1 week \\
7. Minor (occasional) signs \\
8. Pseudomonas \\
9. Positive radiograph \\
10. Diabetes mellitus \\
11. Cranial nerve involvement \\
12. Debilitating conditions \\
13. Old age \\
\hline
\end{tabular}

Data was analysed by using software, SPSS version 26. Quantitative measure, like age, was expressed in terms of mean and standard deviation. Qualitative data were expressed as frequency along with percentages. Pearson's Chi-square test was used to ascertain association between gender, clinico-radiological features, diabetic control, histopathological features, and prognosis of the disease, keeping $p$-value $<0.05$ as significant.

\section{RESULTS}

Twenty-eight patients fulfilled the inclusion criteria, in four years (2016-2019). Out of 28 patients, 17 were males (60.7\%) and 11 females (39.3\%). The mean age of presentation was $59.36 \pm 4.4$ years. Association between gender and survival showed that $41.2 \%$ (7) males and $27.3 \%$ (3) females survived the disease; while $23.8 \%(04)$ males and one (9.1\%) female expired within six months of diagnosis. In $11.8 \%$ (2) males and $27.3 \%$ (3) females, the disease remained static $(p=0.340)$.

Thinning of temporal bone $(2 / 5=40 \%)$ was common among the expired patients. Other grave signs were sclerosis, erosion (mastoid bone), and inflammatory soft tissue in external auditory meatus ( $1 / 5=20 \%$ each). Nine out of $10(90 \%)$ patients that showed inflammatory tissue only, showed improvement in symptoms $(p=0.239)$. Lower motor neuron type facial paralysis of varying degree was seen in 17 (60.7\%) patients (Table II). Pseudomonas aeruginosa was isolated in 14 (50\%), Staphylococcus aureus in 7 (25\%), fungus in 3 (10.7\%) (Aspergillus fumigatus-2 $(7.1 \%)$ and Candida-1 (3.6\%) species and no growth was isolated in 4 (14.3\%) patients. Histopathology reports of ear biopsies showed granulation tissue in $24 / 28$ (85.71\%), nons-pecific inflammation in $3 / 28$ (10.7\%), and squamous cell carcinoma in $1 / 28$ (3.6\%) cases. Association between biopsy reports and survival showed that $20 \%(1 / 5)$ patients diagnosed with squamous cell carcinoma and $80 \%(4 / 5)$ with granulation tissue (GT), expired within six months ( $p=0.543)$, while $90 \%(9 / 10)$ patients with GT and 10\% (1/10) improved. Cultures of ear biopsies revealed that Aspergillus $(2 / 5=40 \%)$ and Pseudomonas $(1 / 5=20 \%)$ were commonest among the expired patients, while staphylococcal $(6 / 10=60 \%)$ and pseudomonal $(3 / 10=30 \%)$ infections were predominant among the improved patients $(p=0.005)$. Association between hearing loss and survival revealed that $10 / 10(100 \%)$ that improved had mild to moderate hearing loss $(p<0.001)$. While $80 \%$ of the expired patients had $(4 / 5)$ severe to profound hearing loss, and only $20 \%$ (1/5) had mild to moderate loss.

Twenty-four out of 28 (85.7\%) patients were diabetics; and out of those 24,18 (75\%) had suboptimal control of disease with $\mathrm{HbAlC}$ levels of more than $7 \%$. Other $4 / 28(14.3 \%)$ had anemia, history of renal transplant and hepatitis $\mathrm{C}$ infection. Among expired group, $80 \%(4 / 5)$ had HBAlc of more than 7 , and $20 \%(1 / 5)$ had good control of diabetes; while, $30 \%$ (3/10) of improved patients had Hbalc $<7$ and 70\% (7/10) had uncontrolled diabetes $(p=0.026$. Tablell).

\section{DISCUSSION}

NOE is a necrotising and destructive infection of the external auditory canal, temporal bone of skull base, and adjacent soft tissues. This study included 28 patients fulfilling the inclusion criteria and presenting in last four years (2016-2019). The gender and the mean age of presentation was similar to the findings by Musa, who found $60.9 \%$ males and $39.1 \%$ females with ratio of $M / F$ 1.5:1. ${ }^{9}$ However, there wasa marked difference of mean age and gender distribution in other studies. ${ }^{10}$

In this study, association between radiological diagnosis/CT findings and survival (six months) revealed that thinning of temporal bone was common among expired patients. Other grave signs were sclerosis, erosion (mastoid bone), and inflammatory soft tissue in external auditory meatus. Patients who showed inflammatory tissue only, showed marked improvement in symptoms. Skull-base osteomyelitis is a serious complication of NOE, which starts as a soft tissue (bacterial/fungal) infection in the EAC (external auditory canal). Then, it spreads through the Santorini fissures and the tympano-mastoid suture to the base of skull. As the disease progresses, it involves the jugular foramen and hypoglossal canal, which carries glossopharyngeal, vagus, spinal accessory, and hypoglossal nerves that also get involved in the spreading infection. ${ }^{11}$ 
Table II: Assciation of gender, risk factors, facial paralysis, CT findings, biopsy, culture, hearing loss, and HbAlc with outcome and prognosis over a period of six months (Renal T: renal transplant, Staph: staphylococcus, Infl: inflammatory).

\begin{tabular}{|c|c|c|c|c|c|c|c|}
\hline \multicolumn{2}{|c|}{ Qualitative variables } & \multirow{2}{*}{$\begin{array}{c}\begin{array}{c}\text { Improved } \\
\mathbf{n}=10\end{array} \\
\mathrm{n}(\%) \\
\end{array}$} & \multirow{2}{*}{$\begin{array}{c}\begin{array}{c}\text { Static } \\
\mathrm{n}=5\end{array} \\
\mathrm{n}(\%) \\
\end{array}$} & $\begin{array}{c}\begin{array}{c}\text { Expired } \\
n=5\end{array} \\
n(\%) \\
\end{array}$ & $\begin{array}{c}\begin{array}{c}\text { Debridement } \\
n=4\end{array} \\
n(\%) \\
\end{array}$ & \multirow{2}{*}{$\begin{array}{c}\begin{array}{c}\text { Lost to FU } \\
n=4\end{array} \\
n(\%) \\
\end{array}$} & \multirow{2}{*}{$\begin{array}{l}\begin{array}{l}\text { Total } \\
\text { n=28 }\end{array} \\
\mathbf{n}(\%) \\
\end{array}$} \\
\hline \multirow{3}{*}{$\begin{array}{l}\text { Gender } \\
p=0.340\end{array}$} & & & & & & & \\
\hline & Males & $7(70)$ & $2(40)$ & $4(80)$ & $1(25)$ & $3(75)$ & $17(60.7)$ \\
\hline & Females & $3(30)$ & $3(60)$ & $1(20)$ & $3(75)$ & $1(25)$ & $11(39.3)$ \\
\hline \multirow{4}{*}{$\begin{array}{l}\text { Risk factors } \\
p=0.39\end{array}$} & Diabetes & $9(90)$ & $5(100)$ & $5(100)$ & $1(25)$ & $4(100)$ & $24(85.7)$ \\
\hline & Anemia & 0 & 0 & 0 & $1(25)$ & 0 & $1(3.57)$ \\
\hline & Renal T & $1(10)$ & 0 & 0 & 0 & 0 & $1(3.57)$ \\
\hline & Hep C & 0 & 0 & 0 & $2(50)$ & 0 & $2(7.14)$ \\
\hline \multirow{5}{*}{$\begin{array}{l}\text { Facial paralysis } \\
p=0.132\end{array}$} & Score II & $3(30)$ & $2(40)$ & 0 & 0 & $2(50)$ & $7(25)$ \\
\hline & Score III & $1(10)$ & $2(40)$ & $1(20)$ & $1(25)$ & 0 & $5(17.9)$ \\
\hline & Score IV & 0 & 0 & $2(40)$ & 0 & $1(25)$ & $3(10.7)$ \\
\hline & Score V & 0 & 0 & $1(20)$ & 0 & $1(25)$ & $2(7.14)$ \\
\hline & No FP & $6(60)$ & $1(20)$ & $1(20)$ & $3(75)$ & 0 & $11(39.3)$ \\
\hline \multirow{4}{*}{$\begin{array}{l}\text { CT findings } \\
p=0.239\end{array}$} & Infl. mass & $9(90)$ & $3(60)$ & $1(20)$ & $4(100)$ & $3(75)$ & $20(71.4)$ \\
\hline & Thinning & 0 & $2(40)$ & $2(40)$ & 0 & $1(25)$ & $5(17.9)$ \\
\hline & Sclerosis & $1(10)$ & 0 & $1(20)$ & 0 & 0 & $2(7.14)$ \\
\hline & Erosion & 0 & 0 & $1(20)$ & 0 & 0 & $1(3.57)$ \\
\hline \multirow{3}{*}{$\begin{array}{l}\text { Biopsy report } \\
p=0.543\end{array}$} & Granulation & $9(90)$ & $4(80)$ & $4(80)$ & $3(75)$ & $4(100)$ & $24(85.7)$ \\
\hline & Nonspecific & $1(10)$ & $1(20)$ & 0 & $1(25)$ & 0 & $3(10.7)$ \\
\hline & $\mathrm{Scc}$ & 0 & 0 & $1(20)$ & 0 & 0 & $1(3.57)$ \\
\hline \multirow{5}{*}{$\begin{array}{l}\text { Culture } \\
p=0.005\end{array}$} & Pseudomonas & $3(30)$ & $5(100)$ & $1(20)$ & $4(100)$ & $1(25)$ & $14(50)$ \\
\hline & Staph. & $6(60)$ & 0 & 0 & 0 & $1(25)$ & $7(25)$ \\
\hline & Aspergillus & 0 & 0 & $2(40)$ & 0 & 0 & $2(7.14)$ \\
\hline & Candida & 0 & 0 & 0 & 0 & $1(25)$ & $1(3.57)$ \\
\hline & None & $1(10)$ & 0 & $2(40)$ & 0 & $1(25)$ & $4(14.3)$ \\
\hline \multirow{2}{*}{$\begin{array}{l}\text { Hearing loss } \\
p<0.001\end{array}$} & Mild to Mod & $10(100)$ & $5(100)$ & $1(20)$ & $4(100)$ & $4(100)$ & $24(85.7)$ \\
\hline & Severe & 0 & 0 & $4(80)$ & 0 & 0 & $4(14.3)$ \\
\hline \multirow{2}{*}{$\begin{array}{l}\mathrm{HbAlc} \\
\mathrm{p}=0.026\end{array}$} & $<7$ & $3(30)$ & 0 & $1(20)$ & $4(100)$ & $2(50)$ & $10(35.7)$ \\
\hline & $>7$ & $7(70)$ & $5(100)$ & $4(80)$ & 0 & $2(50)$ & $18(64.3)$ \\
\hline
\end{tabular}

The disease spreads to the centre and base of skull through four channels: external auditory canal, stylomastoid foramen, jugular foramen, and the apex of the petrous bone. CT and MRI findings are usually helpful in diagnosing NOE at admission, but do not specifically predict the outcome or survival of the disease. ${ }^{12}$

Association between biopsy reports and survival showed that $20 \%$ (5) patients diagnosed with squamous cell carcinoma and $80 \%$ with granulation tissue (GT) expired; while, 90\% (9/10) patients with GT improved. Aspergillius (20\%) and Pseudomonas (20\%) were commonest among expired patients, while Staphylococcal (60\%) and Pseudomonal (30\%) infections were predominant among improved patients $(p=0.005)$. This is in contrast with the findings by Musa, who reported complete improvement in $91 \%$ of infected patients (with Klebsiella and Pseudomonas). ${ }^{9}$ Many studies have shown that Pseudomonas aeruginosa is the most common microbiological organism isolated in culture specimens. Shavit et al. reported that Pseudomonas aeruginosa was the causative agent in $39(44.3 \%)$ of 88 patients and Staphylococcus aureus in $7(8 \%)$ patients. ${ }^{13}$ Bhat et al. found Pseudomonas aeruginosa in 11 (73\%) of total 15 patients. $^{8}$ Similarly, Pseudomonas aeruginosa was isolated from $50 \%$ of 28 swab cultures of the external auditory canal pus. S. aureus, A fumigatus, and Candida were identified in the rest of the patients.

Longstanding DM (diabetes mellitus) causes macrovascular and microvascular angiopathy, which results in suppressed microcirculation, decreased neutrophilic function, chemotaxis, adherence to endothelium, phagocytosis and microbicidal activity along with impaired cytokine production from macrophages. ${ }^{14}$ Macrovascular changes and atherosclerosis cause hypoperfusion, which markedly reduces the transport of antibiotics to the infected area and thus effectiveness of antibiotic therapy. Rigorous control of blood glucose levels and administration of multiple specific antibiotics are essential for treating NOE and preventing complications. In this study, 24 out of $28(85.7 \%)$ patients were diabetics and out of those 24 , $18(75 \%)$ had suboptimal control of disease with $\mathrm{HbAlc}$ levels of more than $7 \%$. Among the expired group, $80 \%$ (4/5) had HBAlc of more than 7 and $20 \%$ had good control of diabetes; while, $30 \%$ of theimproved patients had $\mathrm{Hbalc}<7$ and $70 \%$ $(7 / 10)$ had uncontrolled Diabetes). This is similar to findings by Schwam, who observed uncomplicated diabetes in $42.1 \%$ and complicated diabetes in $17.8 \%$ cases. ${ }^{15}$ Although they also 
stated that Diabetes is not independent risk factor for any outcome, which was not coherent with the present findings Strict control of Diabetes is associated with better survival outcome and less hospital stay time. ${ }^{11,16}$

In this study, those patients who improved, had mild to moderate hearing loss; while, $80 \%$ of the expired patients had severe to profound hearing loss and only $20 \%$ had mild to moderate loss. Other studies did not find any improvement in hearing loss after treatment. ${ }^{19}$ This could be due to lesser number of patients observed than in this study. Mardassi observed improvement in facial paralysis and hearing loss in $75 \%$ cases after administering hyperbaric oxygen therapy and recurrence occurred in $14.28 \%$ of cases $^{17}$

SinceNOE is not specifically a surgical disease, incisional biopsy is done to obtain adequate pus cultures, debridement of necrotic/dead tissue, or to rule out presence of an underlying malignant neoplasm ${ }^{5}$. Surgical debridement is considered only an option, if medical management fails to treat infection. ${ }^{18}$ NOE had a significant mortality rate, approximately up to $50 \%$ by some authors. ${ }^{11,12}$ Disease outcome has improved markedly after the introduction of quinolones for NOE, like ciprofloxacin and other antipseudomonal agents, in the early 1990's ${ }^{2}$. Only early and effective treatment can result in improved prognosis, reduced neurological involvement, and decreased mortality. ${ }^{19}$ In this study, surgical debridement was done in $14.3 \%$ patients. After administering specific therapy, 35.7\% patients improved and were discharged, $17.85 \%$ patients expired, or showed no improvement, in symptomatology despite adequate management of the disease. The rest (14.3\%) were lost to follow-up.

Mortality in NOE is associated with multiple risk factors like immunosuppression (anemia, transplants, hepatitis C, diabetes), local invasion of meninges or brain parenchyma, vascular involvement of carotid arteries resulting in ischemic thromboembolic stroke and aneurysm. ${ }^{20}$ In this study, all the three patients who had anemia and hepatitis $C$ infection expired. However, patients' with renal transplant and immunosuppression improved after treatment (Table II). Similarly, 60\% with advanced cranial nerve involvement expired. Thus, indicating the aggressiveness of the disease after cranial involvement.

\section{CONCLUSION}

This study showed a rising community-acquired NOE with Staphylococcus aureus and less with Pseudomonas infection than previously reported. Fungal infection is associated with poor survival and death, thus requiring aggressive management. Optimal control of Diabetes is the main factor in disease progression to skull-base and other complications. Thinning or sclerosis of temporal bone on $\mathrm{CT}$, uncontrolled Diabetes, sever hearing loss, and facial paralysis score $\mathrm{V} / \mathrm{VI}$, are associated with adverse outcome.

\section{ETHICAL APPROVAL:}

Study was approved by Institutional review board of King Edward Medical University, Lahore, Pakistan (approval letter number: 68/RC/KEMU, dated 16/01/2020).

\section{PATIENTS' CONSENT:}

Since this study was retrospective, consent was not taken.

\section{CONFLICT OF INTEREST:}

Authors declared no conflict of interest.

\section{AUTHORS' CONTRIBUTION:}

KC: Data interpretation.

NA: Critical review.

NR: Manuscript writing.

SQ: Data collection.

SA: Data analysis.

\section{REFERENCES}

1. Chandler JR. Malignant external otitis. Laryngoscope 1968; 78(8):1257-94.

2. Lee SK, Lee SA, Seon SW, Jung JH, Lee JD, Choi JY, et al. Analysis of prognostic factors in malignant external otitis. Clin Exp otorhinolaryngol 2017; 10(3):228-35.

3. Benecke Jr JE. Management of osteomyelitis of the skull base. Laryngoscope 1989; 99(12):1220-3.

4. Orji FT, Akpeh JO, Ukaegbe OC. Malignant otitis externa: An assessment of emerging pathogens and the prognostic factors. Int J Med Med Sci 2017; 9(7):86-91.

5. Glikson E, Sagiv D, Wolf M, Shapira Y. Necrotizing otitis externa: diagnosis, treatment, and outcome in a case series. Diagn Microbiol Infect Dis 2017; 87(1):74-8.

6. Ahmed M, Syed R, More YI, Basha SI. Stenotrophomonas skull base osteomyelitis presenting as necrotizing otitis externa: Unmasking by $\mathrm{CT}$ and MRI-case report and review. Radiol case rep 2019; 14(10):1241-5.

7. Rubin J, Victor LY. Malignant external otitis: Insights into pathogenesis, clinical manifestations, diagnosis, and therapy. $\mathrm{Am}$ J Med 1988; 85(3):391-8.

8. Bhat V, Aziz A, Bhandary SK, Aroor R, Kamath P, Shrinath D, et al. Malignant otitis externa-a retrospective study of 15 patients treated in a tertiary healthcare centre. J Int Adva Otol 2015; 11(1):72-6

9. Musa TS, Bemu AN, Grema US, Kirfi AM. Pattern of otitis externa in Kaduna Nigeria. Pan Afr Med J 2015; 21:165.

10. Abu Eta R, Gavriel H, Stephen K, Eviatar E, Yeheskeli E. The significance of tissue biopsy for fungi in necrotizing otitis externa. Eur Arch Otorhinolaryngol 2018; 275(12):2941-5.

11. Kaya I, Sezgin B, Eraslan S, Öztürk K, Göde S, Bilgen C, et al. Malignant otitis externa: A retrospective analysis and treatment outcomes. Turk Arch Otorhinolaryngol 2018; 56(2):106-10.

12. Nadol Jr JB. Histopathology of pseudomonas osteomyelitis of the temporal bone starting as malignant external otitis. Am J Otolaryngol 1980; 1(5):359-71.

13. Shavit SS, Soudry E, Hamzany Y, Nageris B. Malignant external otitis: factors predicting patient outcomes. Am J Otolaryngol 2016; 37(5):425-30.

14. Cheema KM, Amjad M, Ayub T, Ahmad MM, llyas K, Arsalan D. Skull base osteitis: our experience and systematic review of literature. Esculapio 2013; 9(3):131-6.

15. Schwam ZG, Ferrandino R, Kaul VZ, Wanna GB, Cosetti MK. Thirty-day readmission and prolonged length of stay in malig- 
nant otitis externa. Laryngoscope 2019. doi: 10.1002/ lary.28409. [Online ahead of print].

16. Wiegand S, Berner R, Schneider A, Lundershausen E, Dietz A. Continuing medical education. Otitis externa investigation and evidence-based treatment. Dtsch Arztebl Int 2019; 116(13):224-34.

17. Mardassi A, Turki S, Lahiani R, Mbarek H, Benzarti S, Gharsallah $\mathrm{H}$. Is there a real benefit of hyperbaric oxygeno-therapy in the treatment of necrotizing otitis externa? Tunis Med 2016; 94(12):863.
18. Hatch JL, Bauschard MJ, Nguyen SA, Lambert PR, Meyer TA, McRackan TR. Malignant otitis externa outcomes: A study of the university healthsystem consortium database. Ann Otol Rhinolo 2018; 127(8):514-20.

19. Peled C, Parra A, El-Saied S, Kraus M, Kaplan DM. Surgery for necrotizing otitis externa-indications and surgical findings. Eur Arch Otorhinol 2020; 277(5):1327-34.

20. AlEnazi AS, Al Sharhan SS, Telmesani LM, Aljazan NA, Al Qahtani BM, Lotfy MA. The impact of using the term "diabetic ear" for the patients with skull base osteomyelitis. J Family Community Med 2019; 26(1):23-9 